Georgian Mathematical Journal

Volume 12 (2005), Number 4, 595-606

\title{
ON A GENERALIZED WILSON FUNCTIONAL EQUATION
}

\author{
ROMAN BADORA
}

\begin{abstract}
We solve the functional equation

$$
\sum_{n=0}^{N-1} g\left(x+k_{n} \cdot y\right)=N g(x) f(y), \quad x, y \in G
$$

where $G$ is a locally compact Abelian group, $\left\{k_{0}=i d_{G}, k_{1}, \ldots, k_{N-1}\right\}$ is a finite group of continuous automorphisms of $G, f$ is a bounded continuous complex-valued function on $G$ and $g$ is an almost periodic function on $G$.
\end{abstract}

2000 Mathematics Subject Classification: 39B32.

Key words and phrases: Wilson functional equation, spherical function.

\section{INTRODUCTION}

Let $(G,+)$ be a locally compact Abelian group, $N \in \mathbb{N}$ and let $K=\left\{k_{0}=\right.$ $\left.i d_{G}, k_{1}, \ldots, k_{N-1}\right\}$ be a finite group of continuous automorphisms of $G$ (the action of $k \in K$ on $x \in G$ is denoted by $k \cdot x$ ).

In this paper we find the set of solutions of the following version of Wilson's functional equation:

$$
\sum_{n=0}^{N-1} g\left(x+k_{n} \cdot y\right)=N g(x) f(y)
$$

where $f: G \rightarrow \mathbb{C}$ is a bounded continuous function and $g: G \rightarrow \mathbb{C}$ is an almost periodic function. This functional equation was studied by Förg-Rob and Schwaiger [4], Gajda [6] and Stetkær [13], [14]. In the main the authors found only the form of the function $f$. Our aim is to calculate also the form of the function $g$, which combined with the results by Stetkær [12] leads to a solution of the Pexiderized version (6) of (1) and of more general functional equations like (14) and (15). However, like [5], but in contrast to the works just mentioned, we do not seek our solutions among all continuous functions or even among the bounded continuous functions, but in the subalgebra of all almost periodic functions on $G$. We find that to each such solution there corresponds a character $\gamma \in \hat{G}$ ( $\hat{G}$ is defined on the next page) such that the solution is a linear combination of the functions $x \rightarrow \gamma(k \cdot x)$, where $k \in K$. This is known for the function $f$ in (1), but not for $g$ for a general group $K$.

In the case where $G=\mathbb{C}$ and $\omega$ is an $N^{\text {th }}$ root of 1 such that $\omega^{n} \neq 1$ for $n=1,2, \ldots, N-1$, equation (1) with $K=\left\{1, \omega, \omega^{2}, \ldots, \omega^{N-1}\right\}$ reduces to the 
equation

$$
\sum_{n=0}^{N-1} g\left(x+\omega^{n} \cdot y\right)=N g(x) f(y),
$$

which was solved by Stetkær in [14].

The classical example of (1) is Wilson's first generalization of d'Alembert's functional equation (see Wilson [15] and Aczél [1])

$$
g(x+y)+g(x-y)=2 g(x) f(y),
$$

while $K=\left\{i d_{G},-i d_{G}\right\}$.

In the final part of our paper we consider the case where the finite group $K$ is replaced by a compact group.

\section{Almost Periodic Functions}

Let $G$ be a topological group. Then $B_{c}(G)$ denotes the Banach algebra of all continuous complex-valued bounded functions on $G$ with the supremum norm.

If $f \in B_{c}(G)$ and $x \in G$, then by $f_{x}$ we denote the complex-valued function defined on $G$ by the formula

$$
f_{x}(y)=f(y x), \quad y \in G .
$$

A function $f \in B_{c}(G)$ is called almost periodic if the set $\left\{f_{x}: x \in G\right\}$ is relatively compact in $B_{c}(G)$. The set of all almost periodic functions on $G$ with the norm of uniform convergence is a Banach algebra which is denoted by $\mathfrak{A}_{c}(G)$ (see [7], vol. I, Theorem 18.3).

It is known ([7], vol. I, Theorem 18.8) that on the space $\mathfrak{A}_{c}(G)$ there exists a complex linear functional $M$ with the following properties:

(A) $M\left(f_{x}\right)=M(f)$, for $f \in \mathfrak{A}_{c}(G)$ and $x \in G$;

(B) $M(f)>0$, for $f \in \mathfrak{A}_{c}(G)$ with $f \geq 0_{G}, f \neq 0_{G}$;

(C) $M\left(1_{G}\right)=1$.

Such a functional $M$ is continuous (its norm is equal to 1), uniquely determined (see [7], vol. I, Theorem 18.9) and referred to as the invariant mean on the space $\mathfrak{A}_{c}(G)$.

A continuous homomorphism of $G$ into the complex unit circle is called a character of $G$ (see [7]). The set of all characters of $G$ equipped with the pointwise multiplication forms an Abelian group which we denote by $\hat{G}$. It can be proved easily (using the definition) that all characters are almost periodic.

Finally, we define the Fourier transformation on $\mathfrak{A}_{c}(G)$ by the formula

$$
\hat{f}(\gamma)=M(f \cdot \bar{\gamma}), \quad \gamma \in \hat{G}
$$

for all $f \in \mathfrak{A}_{c}(G)$, where $\bar{\gamma}$ is a character of the group $G$ defined by

$$
\bar{\gamma}(x)=\overline{\gamma(x)}, \quad x \in G \text {. }
$$

If $G$ is a locally compact Abelian group, then $\hat{G}$ is a separating family and then the Fourier transformation is injective and the inversion theorem holds. In particular, we have 
Lemma 1 (see [9], Satz V.31.3). If $G$ is a locally compact Abelian group, $f \in \mathfrak{A}_{c}(G)$ and $f \neq 0_{G}$, then there exists a character $\gamma \in \hat{G}$ such that

$$
\hat{f}(\gamma) \neq 0
$$

Lemma 2 (see [8] or [5], Lemma 3). If $G$ is a locally compact Abelian group, $f \in \mathfrak{A}_{c}(G)$ and $k \in K$, then $f \circ k \in \mathfrak{A}_{c}(G)$.

\section{The Main Result}

Let $f$ and $g$ satisfy equation (1). If $g=0_{G}$, then $f$ is an arbitrary function. If $f=0_{G}$, then putting $y=0$ in (1) we have $g=0_{G}$. So, in the sequel we can assume that $f \neq 0_{G}$ and $g \neq 0_{G}$. (It suffices to assume that $g \neq 0_{G}$, because then also $f \neq 0_{G}$. Indeed, putting $y=e$ in $(1)$ we find that $f(e)=1$.)

Our main result reads as follows

Theorem 1. Let $(G,+)$ be a locally compact Abelian group, $N \in \mathbb{N}$ and let $K=\left\{k_{0}=i d_{G}, k_{1}, \ldots, k_{N-1}\right\}$ be a finite group of continuous automorphisms of $G$. Then functions $f \in B_{c}(G) \backslash\{0\}$ and $g \in \mathfrak{A}_{c}(G) \backslash\{0\}$ satisfy equation (1) if and only if there exist a character $\gamma \in \hat{G}$ and a function $\kappa: K \rightarrow \mathbb{C}$ such that

$$
\begin{gathered}
f(x)=\frac{1}{N} \sum_{n=0}^{N-1} \gamma\left(k_{n} \cdot x\right), \quad x \in G \\
g(x)=\frac{1}{N} \sum_{n=0}^{N-1} \kappa\left(k_{n}\right) \gamma\left(k_{n} \cdot x\right), \quad x \in G .
\end{gathered}
$$

Proof. Simple calculations show that functions of form (2)-(3) satisfy equation (1).

Let $f$ and $g$ satisfy equation (1). Then the function $f$ satisfies the following functional equation (see [12], Corollary III.2):

$$
\sum_{n=0}^{N-1} f\left(x+k_{n} \cdot y\right)=N f(x) f(y),
$$

for all $x, y \in G$.

From equation (1) and Lemma 2 we infer that $f$ is an almost periodic function and Lemma 1 implies the existence of a character $\gamma \in \hat{G}$ such that

$$
\hat{f}(\gamma) \neq 0
$$

Applying equation (4) and the linearity of $M$ we get

$$
\begin{aligned}
N f(y) \hat{f}(\gamma) & =N f(y) M_{x}(f(x) \overline{\gamma(x)}) \\
& =M_{x}(N f(y) f(x) \overline{\gamma(x)}) \\
& =M_{x}\left(\sum_{n=0}^{N-1} f\left(x+k_{n} \cdot y\right) \overline{\gamma(x)}\right), \quad y \in G,
\end{aligned}
$$


(in the above formula and in the sequel the subscript letter next to $M$ shows that $M$ is applied to a function of the respective variable) which, jointly with the linearity of $M$ and property (A), yields

$$
\begin{aligned}
N f(y) \hat{f}(\gamma) & =\sum_{n=0}^{N-1} M_{x}\left(f\left(x+k_{n} \cdot y\right) \overline{\gamma\left(x+k_{n} \cdot y-k_{n} \cdot y\right)}\right) \\
& =\sum_{n=0}^{N-1} M_{x}\left(f(x) \overline{\gamma\left(x-k_{n} \cdot y\right)}\right) \\
& =\sum_{n=0}^{N-1} M_{x}\left(f(x) \overline{\gamma(x)} \gamma\left(k_{n} \cdot y\right)\right) \\
& =\sum_{n=0}^{N-1} \hat{f}(\gamma) \gamma\left(k_{n} \cdot y\right), \quad y \in G .
\end{aligned}
$$

Hence, by (5), we have

$$
f(x)=\frac{1}{N} \sum_{n=0}^{N-1} \gamma\left(k_{n} \cdot x\right), \quad x \in G,
$$

which is the required formula for $f$.

Similarly, using equation (1) and the assumption that $g \in \mathfrak{A}_{c}(G)$, for each $x \in G$, we get

$$
N g(x) \hat{f}(\gamma)=\sum_{n=0}^{N-1} M_{y}\left(g\left(k_{n} \cdot y\right) \overline{\gamma(y)}\right) \gamma\left(k_{n}^{-1} \cdot x\right)
$$

Putting

$$
\kappa\left(k_{n}^{-1}\right)=\frac{M_{y}\left(g\left(k_{n} \cdot y\right) \overline{\gamma(y)}\right)}{\hat{f}(\gamma)}, \quad n \in\{0,1, \ldots, N-1\},
$$

we define the function $\kappa: K \rightarrow \mathbb{C}$ and now

$$
N g(x) \hat{f}(\gamma)=\sum_{n=0}^{N-1} \kappa\left(k_{n}^{-1}\right) \hat{f}(\gamma) \gamma\left(k_{n}^{-1} \cdot x\right), \quad x \in G .
$$

Dividing the above equation by $N \hat{f}(\gamma)$ we obtain the required formula for the function $g$ and the proof of Theorem 1 is finished.

Remark 1. The character $\gamma$ in (2) (or (3)) is unique modulo $K$ for given $f$ $(g):$ If

$$
f(x)=\frac{1}{N} \sum_{n=0}^{N-1} \delta\left(k_{n} \cdot x\right), \quad x \in G,
$$

for some $\delta \in \hat{G}$, then there exists $k \in K$ such that $\delta=\gamma \circ k$. This follows from the linear independence of the set of characters (see [7], Lemma 29.41). 
Remark 2. Chojnacki in [3] proved that any non-zero bounded continuous solution of (4) has the form

$$
f(x)=\frac{1}{N} \sum_{n=0}^{N-1} \delta\left(k_{n} \cdot x\right), \quad x \in G,
$$

for some $\delta \in \hat{G}$. Thus $f$ is almost periodic. So, this fact follows without using the assumption that $g$ is almost periodic.

\section{Examples of the Application}

For the Pexider version of equation (1)

$$
\sum_{n=0}^{N-1} g\left(x+k_{n} \cdot y\right)=N h(x) f(y), \quad x, y \in G,
$$

we have

Corollary 1. Let $(f, g, h)$ be a solution of (6) such that $f, h \in B_{c}(G) \backslash\left\{0_{G}\right\}$ and $g \in \mathfrak{A}_{c}(G) \backslash\left\{0_{G}\right\}$. Then there exist a character $\gamma \in \hat{G}$, a function $\kappa: K \rightarrow \mathbb{C}$ and a constant $c \in \mathbb{C} \backslash\{0\}$ such that

$$
\begin{aligned}
& f(x)=c \frac{1}{N} \sum_{n=0}^{N-1} \gamma\left(k_{n} \cdot x\right), \quad x \in G ; \\
& g(x)=\frac{1}{N} \sum_{n=0}^{N-1} \kappa\left(k_{n}\right) \gamma\left(k_{n} \cdot x\right), \quad x \in G ; \\
& h(x)=\frac{1}{c} \frac{1}{N} \sum_{n=0}^{N-1} \kappa\left(k_{n}\right) \gamma\left(k_{n} \cdot x\right), \quad x \in G .
\end{aligned}
$$

Conversely, $(f, g, h)$ given by (7), (8) and (9) solves (6).

Proof. Putting $y=0$ in (6) we have

$$
g(x)=h(x) f(0), \quad x \in G .
$$

Since $g$ is a non-zero function, we have

$$
f(0) \neq 0
$$

and condition (10) implies

$$
h(x)=\frac{1}{f(0)} g(x), \quad x \in G .
$$

The function $g$ and the function $f_{1}: G \rightarrow \mathbb{C}$ defined by the formula

$$
f_{1}(x)=\frac{1}{f(0)} f(x), \quad x \in G,
$$


satisfy equation (1). From Theorem 1 we obtain the existence of a character $\gamma \in \hat{G}$ and a function $\kappa: K \rightarrow \mathbb{C}$ such that

$$
\frac{1}{f(0)} f(x)=\frac{1}{N} \sum_{n=1}^{N-1} \gamma\left(k_{n} \cdot x\right), \quad x \in G,
$$

and

$$
g(x)=\frac{1}{N} \sum_{n=1}^{N-1} \kappa\left(k_{n}\right) \gamma\left(k_{n} \cdot x\right), \quad x \in G .
$$

Putting $c=f(0)$ and using (11), (12) and (13) we get the required formulas for $f, g$ and $h$.

Now we shall study the extension of equation (1)

$$
\sum_{n=0}^{N-1} g\left(x+k_{n} \cdot y\right) \overline{\chi\left(k_{n}\right)}=N g(x) f(y), \quad x, y \in G,
$$

and the equation

$$
\sum_{n=0}^{N-1} g\left(x+k_{n} \cdot y\right) \overline{\chi\left(k_{n}\right)}=N f(x) g(y), \quad x, y \in G,
$$

where $\chi: K \rightarrow \mathbb{C}$ is a given character of the group $K$.

Equations (14) and (15) were studied by Stetkær in [12]. The classical examples of (14) and (15) are

$$
g(x+y)-g(x-y)=2 g(x) f(y)
$$

and

$$
g(x+y)-g(x-y)=2 f(x) g(y)
$$

while $K=\left\{i d_{G},-i d_{G}\right\}$ and $\chi$ is defined by

$$
\chi\left(i d_{G}\right)=1 \text { and } \chi\left(-i d_{G}\right)=-1 .
$$

Proposition 1. Let $(G,+)$ be a locally compact Abelian group, $N \in \mathbb{N}$, $K=\left\{k_{0}=i d_{G}, k_{1}, \ldots, k_{N-1}\right\}$ be a finite group of continuous automorphisms of $G$ and let $\chi \in \hat{K}$ be a character of $K$. Assume that $(f, g, h)$ is a solution of the functional equation

$$
\sum_{n=0}^{N-1} g\left(x+k_{n} \cdot y\right) \overline{\chi\left(k_{n}\right)}=N h(x) f(y), \quad x, y \in G,
$$

satisfying $g \in B_{c}(G) \backslash\left\{0_{G}\right\}$ and $f, h \in \mathfrak{A}_{c}(G) \backslash\left\{0_{G}\right\}$. Then there exist a character $\gamma \in \hat{G}$ and functions $\iota, \kappa: K \rightarrow \mathbb{C}$ such that

$$
f(x)=\frac{1}{N} \sum_{n=0}^{N-1} \iota\left(k_{n}\right) \gamma\left(k_{n} \cdot x\right), \quad x \in G,
$$


and

$$
h(x)=\frac{1}{N} \sum_{n=0}^{N-1} \kappa\left(k_{n}\right) \gamma\left(k_{n} \cdot x\right), \quad x \in G .
$$

Proof. The proof of Stetkær's result ([12], Theorem III.1) guarantees the existence of a non-zero bounded continuous function $F: G \rightarrow \mathbb{C}$ such that

$$
\sum_{n=0}^{N-1} h\left(x+k_{n} \cdot y\right)=N h(x) F(y), \quad x, y \in G,
$$

and

$$
\sum_{n=0}^{N-1} \check{f}\left(x+k_{n} \cdot y\right)=N \check{f}(x) \check{F}(y), \quad x, y \in G,
$$

where $\check{f}$ and $\check{F}$ are defined as follows:

$$
\check{f}(x)=f(-x) \text { and } \check{F}(x)=(-x), \quad x \in G .
$$

In [5] Gajda proved (Lemma 3) that if a function $\varphi \in \mathfrak{A}_{c}(G)$ and $m$ is a morphism of the group $G$, then $\varphi \circ m \in \mathfrak{A}_{c}(G)$ and

$$
M(\varphi \circ m)=M(\varphi) \text {. }
$$

Therefore

$$
\check{f} \in \mathfrak{A}_{c}(G) \backslash\left\{0_{G}\right\}
$$

(see also [9], Satz II.7.3). Applying Theorem 1 to equation (17) we have the existence of a character $\gamma \in \hat{G}$ and a function $\kappa: K \rightarrow \mathbb{C}$ such that

$$
\begin{gathered}
\hat{F}(\gamma) \neq 0, \\
F(x)=\frac{1}{N} \sum_{n=0}^{N-1} \gamma\left(k_{n} \cdot x\right), \quad x \in G,
\end{gathered}
$$

and

$$
h(x)=\frac{1}{N} \sum_{n=0}^{N-1} \kappa\left(k_{n}\right) \gamma\left(k_{n} \cdot x\right), \quad x \in G .
$$

Then

$$
\begin{aligned}
\check{F}(x) & =F(-x)=\frac{1}{N} \sum_{n=0}^{N-1} \gamma\left(k_{n} \cdot(-x)\right) \\
& =\frac{1}{N} \sum_{n=0}^{N-1} \gamma\left(k_{n} \cdot(-x)\right)=\frac{1}{N} \sum_{n=0}^{N-1} \overline{\gamma\left(k_{n} \cdot x\right)} \\
& =\frac{1}{N} \sum_{n=0}^{N-1} \bar{\gamma}\left(k_{n} \cdot x\right), \quad x \in G .
\end{aligned}
$$


Moreover, using Gajda's Lemma, we get

$$
\begin{aligned}
(\check{F})^{\gamma}(\bar{\gamma}) & =M_{x}(\check{F}(x) \overline{\bar{\gamma}(x)}) \\
& =M_{x}(F(-x) \overline{\gamma(-x)})=M_{x}(F(x) \overline{\gamma(x)}) \\
& =\hat{F}(\gamma) \neq 0 .
\end{aligned}
$$

Repeating the adequate part of the proof of Theorem 1 for equation (18) we derive the form of $\check{f}$

$$
\check{f}(x)=\frac{1}{N} \sum_{n=0}^{N-1} \iota\left(k_{n}\right) \bar{\gamma}\left(k_{n} \cdot x\right), \quad x \in G,
$$

with some function $\iota: K \rightarrow \mathbb{C}$, which means that

$$
f(x)=\frac{1}{N} \sum_{n=0}^{N-1} \iota\left(k_{n}\right) \gamma\left(k_{n} \cdot x\right), \quad x \in G,
$$

and ends the proof of Proposition 1.

As a deduction from this proposition we obtain

Corollary 2. Let $\chi \in \hat{K}$ and let $f, g \in \mathfrak{A}_{c}(G) \backslash\left\{0_{G}\right\}$ satisfy equation (14). Then there exist a character $\gamma \in \hat{G}$ and functions $\iota, \kappa: K \rightarrow \mathbb{C}$ such that

$$
f(x)=\frac{1}{N} \sum_{n=0}^{N-1} \iota\left(k_{n}\right) \gamma\left(k_{n} \cdot x\right), \quad x \in G,
$$

and

$$
g(x)=\frac{1}{N} \sum_{n=0}^{N-1} \kappa\left(k_{n}\right) \gamma\left(k_{n} \cdot x\right), \quad x \in G .
$$

and

Corollary 3. Let $\chi \in \hat{K}$ and let $f, g \in \mathfrak{A}_{c}(G) \backslash\left\{0_{G}\right\}$ satisfy equation (15). Then there exist a character $\gamma \in \hat{G}$ and functions $\iota, \kappa: K \rightarrow \mathbb{C}$ such that

$$
f(x)=\frac{1}{N} \sum_{n=0}^{N-1} \iota\left(k_{n}\right) \gamma\left(k_{n} \cdot x\right), \quad x \in G,
$$

and

$$
g(x)=\frac{1}{N} \sum_{n=0}^{N-1} \kappa\left(k_{n}\right) \gamma\left(k_{n} \cdot x\right), \quad x \in G .
$$




\section{The Case of a Compact Group $K$}

In this section we assume that $K$ is a compact subgroup of the group $\operatorname{Aut}(G)$ of all automorphisms of $G$ onto $G$ with the normalized Haar measure $\mu$ (see also [7]). So $K$ needs no longer be finite. The functional equation (1) generalizes to

$$
\int_{K} g(x+k \cdot y) d \mu(k)=g(x) f(y), \quad x, y \in G .
$$

If $g$ is a non-zero continuous function, then a non-zero solution $f$ of this functional equation is called a $K$-spherical function and then we say that the function $g$ is associated with the function $f$ (see [12]).

Theorem 2. Let $(G,+)$ be a locally compact Abelian group and let $K$ be a compact subgroup of the group $\operatorname{Aut}(G)$ with the normalized Haar measure $\mu$. Moreover, suppose that $f \in B_{c}(G) \backslash\left\{0_{G}\right\}$ and $g: G \rightarrow \mathbb{C}$ is a function such that for each $x \in G$ the map

$$
K \times G \ni(k, y) \longrightarrow g(x+k \cdot y) \in \mathbb{C}
$$

is a member of $\mathfrak{A}_{c}(K \times G) \backslash\left\{0_{G}\right\}$.

If the functions $f$ and $g$ satisfy equation (19) then there exist a character $\gamma \in \hat{G}$ and a continuous function $\kappa: K \rightarrow \mathbb{C}$ such that

$$
f(x)=\int_{K} \gamma(k \cdot x) d \mu(k), \quad x \in G,
$$

and

$$
g(x)=\int_{K} \kappa(k) \gamma(k \cdot x) d \mu(k), \quad x \in G .
$$

Conversely, $(f, g)$ given by (21) and (22) solves (19).

Proof. First we choose $x_{0} \in G$ such that $g\left(x_{0}\right) \neq 0$. Then

$$
f(y)=\frac{1}{g\left(x_{0}\right)} \int_{K} g\left(x_{0}+k \cdot y\right) d \mu(k), \quad y \in G .
$$

A standard calculation (see also [12], Corollary III.2) shows that the function $f$ satisfies the following functional equation:

$$
\int_{K} f(x+k \cdot y) d \mu(k)=f(x) f(y), \quad x, y \in G .
$$

The Fubini Theorem for invariant means on $\mathfrak{A}_{c}(K \times G)$ (see [9], Kapitel II, $\S 12$, Satz 2) and identity (23) imply that $f \in \mathfrak{A}_{c}(G)$ (because the functional

$$
C(K) \ni \varphi \rightarrow \int_{K} \varphi(k) d \mu(k)
$$

is an invariant mean on the space $\mathfrak{A}_{c}(K)=C(K)$ of all continuous functions on $K)$. 
Since $f \neq 0$, one can find a character $\gamma \in \hat{G}$ such that

$$
\hat{f}(\gamma) \neq 0
$$

By (24), for each $y \in G$, we have

$$
\begin{aligned}
f(y) \hat{f}(\gamma) & =f(y) M_{x}(f(x) \overline{\gamma(x)}) \\
& =M_{x}(f(x) f(y) \overline{\gamma(x)}) \\
& =M_{x}\left(\int_{K} f(x+k \cdot y) \overline{\gamma(x)} d \mu(k)\right) .
\end{aligned}
$$

Moreover, we may apply the Fubini Theorem for invariant means on $\mathfrak{A}_{c}(K \times G)$. As a result we get

$$
\begin{aligned}
f(y) \hat{f}(\gamma) & =\int_{K}\left(M_{x}(f(x+k \cdot y) \overline{\gamma(x)}) d \mu(k)\right. \\
& =\int_{K}\left(M_{x}(f(x+k \cdot y) \overline{\gamma(x+k \cdot y-k \cdot y)}) d \mu(k)\right. \\
& =\int_{K}\left(M_{x}(f(x+k \cdot y) \overline{\gamma(x+k \cdot y)} \gamma(k \cdot y)) d \mu(k)\right. \\
& =\int_{K}\left(M_{x}(f(x) \overline{\gamma(x)} \gamma(k \cdot y)) d \mu(k)\right. \\
& =\int_{K} \hat{f}(\gamma) \gamma(k \cdot y) d \mu(k) \\
& =\hat{f}(\gamma) \int_{K} \gamma(k \cdot y) d \mu(k),
\end{aligned}
$$

for all $y \in G$. Dividing this equation by $\hat{f}(\gamma)$ we obtain the required formula for $f$.

Next, using equation (19), assumption (20) and Fubini's Theorem, for each $x \in G$, we get

$$
\begin{aligned}
g(x) \hat{f}(\gamma) & =g(x) M_{y}(f(y) \overline{\gamma(y)}) \\
& =M_{y}(g(x) f(y) \overline{\gamma(y)}) \\
& =M_{y}\left(\int_{K} g(x+k \cdot y) \overline{\gamma(y)} d \mu(k)\right) \\
& =\int_{K}\left(M_{y}(g(x+k \cdot y) \overline{\gamma(y)})\right) d \mu(k) \\
& =\int_{K}\left(M_{y}\left(g\left(k \cdot\left(y+k^{-1} \cdot x\right) \overline{\gamma\left(y+k^{-1} \cdot x-k^{-1} \cdot x\right)}\right)\right) d \mu(k)\right.
\end{aligned}
$$




$$
\begin{aligned}
& =\int_{K}\left(M_{y}\left(g(k \cdot y) \overline{\gamma(y)} \gamma\left(k^{-1} \cdot x\right)\right)\right) d \mu(k) \\
& =\int_{K}\left(M_{y}(g(k \cdot y) \overline{\gamma(y)})\right) \gamma\left(k^{-1} \cdot x\right) d \mu(k) .
\end{aligned}
$$

Putting

$$
\kappa\left(k^{-1}\right)=\frac{M_{y}(g(k \cdot y) \overline{\gamma(y)})}{\hat{f}(\gamma)}, \quad k \in K,
$$

and dividing the above equation by $\hat{f}(\gamma)$ we have the desired form of $g$, which ends the proof.

Arguments similar to those used in the proof of Corollary 1 lead to

Corollary 4. Let $(f, g, h)$ be a solution of the functional equation

$$
\int_{K} g(x+k \cdot y) d \mu(k)=h(x) f(y), \quad x, y \in G,
$$

such that $f, h \in B_{c}(G) \backslash\left\{0_{G}\right\}$ and $g$ satisfies condition (20). Then there exist a character $\gamma \in \hat{G}$, a continuous function $\kappa: K \rightarrow \mathbb{C}$ and a constant $c \in \mathbb{C} \backslash\{0\}$ such that

$$
\begin{aligned}
& f(x)=c \int_{K} \gamma(k \cdot x) d \mu(k), \quad x \in G ; \\
& g(x)=\int_{K} \kappa(k) \gamma(k \cdot x) d \mu(k), \quad x \in G ; \\
& h(x)=\frac{1}{c} \int_{K} \kappa(k) \gamma(k \cdot x) d \mu(k), \quad x \in G .
\end{aligned}
$$

Conversely, $(f, g, h)$ given by (26), (27) and (28) solves (25).

This result was proved by Stetkær ([10], Theorem 3) under the assumption that $G$ is a compact group.

\section{REFERENCES}

1. J. ACzÉL, Vorlesungen über Funktionalgleichungen und ihre Anwendungen. Lehrbücher und Monographien aus dem Gebiete der exakten Wissenschaften, Mathematische Reihe, Bd. 25. Birkhäuser Verlag, Basel-Stuttgart, 1961.

2. R. BADORA, On a joint generalization of Cauchy's and d'Alembert's functional equations. Aequationes Math. 43(1992), No. 1, 72-89.

3. W. Chojnacki, On some functional equation generalizing Cauchy's and d'Alembert's functional equations. Colloq. Math. 55(1988), No. 1, 169-178.

4. W. Förg-Rob and J. SchwAIGER, A generalization of the cosine equation to $n$ summands. Selected topics in functional equations and iteration theory (Graz, 1991), 219-226, Grazer Math. Ber., 316, Karl-Franzens-Univ. Graz, Graz, 1992. 
5. Z. GAJDA, On functional equations associated with characters of unitary representations of groups. Aequationes Math. 44(1992), No. 1, 109-121.

6. Z. GajDA, A remark on the talk of W. Förg-Rob. Selected topics in functional equations and iteration theory (Graz, 1991), 234-237, Grazer Math. Ber., 316, Karl-Franzens-Univ. Graz, Graz, 1992.

7. E. HewitT and K. A. Ross, Abstract harmonic analysis. Vol. I and II. Die Grundlehren der mathematischen Wissenschaften, Band 152. Springer-Verlag, New York-Berlin, 1970.

8. H. HEYER, Probability measures on locally compact groups. Ergebnisse der Mathematik und ihrer Grenzgebiete, Band 94. Springer-Verlag, Berlin-New York, 1977.

9. W. MaAk, Fastperiodische Funktionen. Die Grundlehren der Mathematischen Wissenschaften in Einzeldarstellungen mit besonderer Berücksichtigung der Anwendungsgebiete, Band LXI. Springer-Verlag, Berlin-Göttingen-Heidelberg, 1950.

10. H. Stetkær, Functional equations and spherical functions. Preprint Series 1994 No. 18, Mathematisk Institut, Aarhus University, Denmark, 1-28.

11. H. StetkæR, d'Alembert's equation and spherical functions. Aequationes Math. 48 (1994), No. 2-3, 220-227.

12. H. Stetkær, Wilson's functional equations on groups. Aequationes Math. 49(1995), No. $3,252-275$.

13. H. Stetkær, On a signed cosine equation of $N$ summands. Aequationes Math. 51(1996), No. 3, 294-302.

14. H. StetKær, Wilson's functional equation on C. Aequationes Math. 53(1997), No. 1-2, 91-107.

15. W. H. Wilson, On certain related functional equations. American M. S. Bull. 26(1919), 300-312 (1919-20).

(Received 29.11.2004; revised 5.02.2005)

Author's address:

Institute of Mathematics

Silesian University

Bankowa 14, PL 40-007 Katowice

Poland

E-mail: robadora@ux2.math.us.edu.pl 